\title{
Accessible Wayfinding Solutions for Persons with Disabilities
}

\author{
Mada Center
}

The task of navigating through new spaces can often be challenging for people with disabilities, and especially for people with visual disabilities and the elderly unless they were already acquainted with the pathways and key landmarks. This applies to both indoor and outdoor spaces. To achieve safe and independent mobility, PWD's usually depend on published information, prior experience, the knowledge of others, and/or technology to navigate through unfamiliar outdoor and indoor environments. Today, due to advances in various technologies, wayfinding and navigation systems and services are more common and accessible on all platforms for end-users.

Of the many features that smart cities offer, the safe and comfortable mobility of pedestrians within the built environment is of particular importance. Safe and comfortable mobility requires that the built environments of smart cities be accessible to all pedestrians, mobility abled and mobility impaired, given their various mobility needs and preferences. Through this, coupled with advanced technologies such as wayfinding applications, pedestrians can get assistance in finding the best pathways to use at different locations and times. Wayfinding applications are usually comprised of two components, accessibility data, and appropriate algorithms that can utilize that data to meet the mobility needs and preferences of all individuals.

Accessible wayfinding technology offers great solutions to guide the blind and more generally people with disabilities indoors and outdoors. Public places like metro stations, airports, bus stations, entertainment centers, malls, tourist spots, and much more are now adapting to the inclusive design by relying on indoor and outdoor wayfinding solutions.

\section{Key Highlights of Accessible Wayfinding Solutions}

\section{Seamless Indoor and Outdoor experience}

The wayfinding app must be able to provide seamless routing and navigation between rooms, floors, buildings, and remote areas.

Indoor positioning with Bluetooth Low Energy beacons

The easiest, most cost-effective method with the smallest interference with other IT 
networks is to use commercial matchbox-sized BLE beacons. These beacons are usually attached with double-sided tape, to crucial locations inside buildings, to deliver location services for mobile phones.

\section{Easy Map Editor tools}

It should be easy for users with no IT skills to update digital maps to accommodate small changes and create points of interest, using simple drag and drop gesture.

\section{Integrated solution}

Digital maps and wayfinding solutions should be integrated into one platform.

\section{Kiosk Based WayFinding}

Wayfinding Kiosks with barrier-free building information, tactile maps, sound alerts, sign language services, and auto physical adaptability.

\section{Universal Design}

To develop the designed solutions from a universal design perspective.

\section{Mobile Apps for Accessible Wayfinding}

Mobile apps are designed to work with devices such as smartphones and tablets working on the IOS and Android platforms. Several are designed specifically for people with disabilities, while others are designed for the general public but are accessible to people with disabilities.

Mada successfully has set up the Lazarillo INS App, the winner of Mada Seedstars ICT Accessibility Awards at Mathaf museum as part of improving accessible tourism in Qatar. Lazarillo will digitize floor maps, detailing places of interest (services), which will allow users to create direct routes to their specific desired service. The app will include a narrative about museums' galleries allowing the user to have a guided tour. Lazarillo's indoor positioning technology uses Bluetooth beacons, which are small, autonomous devices that can be detected by smartphones and are easy to install on any surface within a facility.

\section{Accessible Wayfinding Kiosk}

Visiting an unfamiliar destination especially those large public buildings such as shopping centers, university campuses, science museums, etc. can be stressful as it increases the chance where people may get lost at some point. Accessible Kiosks come with wayfinding software to help shoppers or visitors to navigate through the maps and 
Nafath

Issue 17 - May 2021

directories and also find their way to the desired location. This kiosk has the following features:

- Equipped with digital braille and tactile functions for the visually impaired.

- Universal design for the physically disabled.

- Equipped with voice support function for the elderly.

- Real-time Braille, Sign language, audio induction loop, voice guidance for the indoor positioning of the public space. 